EL CHOCOLATE, DE BEBIDA DE NOBLES A GOLOSINA DE TODOS

\title{
EL CHOCOLATE, DE BEBIDA DE NOBLES A GOLOSINA DE TODOS
}

\author{
Deyanira Munguía Ortíz*, Manuela Camacho Gómez**
}

* Licenciada en Relaciones Comerciales. Universidad Juárez Autónoma de Tabasco, México.

** Doctora en Educación Internacional. Profesora-Investigadora de la División Académica de Ciencias Económico Administrativas. Universidad Juárez Autónoma de Tabasco, México.

Dirección para recibir correspondencia: deya_2207@hotmail.com 
EL CHOCOLATE, DE BEBIDA DE NOBLES A GOLOSINA DE TODOS

\section{RESUMEN}

Este trabajo es resultado de una amplia revisión literaria, que siguió un orden estrictamente descriptivo, el procedimiento fue orientado a desarrollar una explicación narrativa sobre el proceso histórico de la difusión y comercialización del chocolate desde la época prehispánica a la actual. Diversos autores dan cuenta de las transformaciones del chocolate, desde el concepto original de bebida mezclada con diferentes especias, la cual arribó a España y de allí a buena parte de Europa, donde le añadieron valor incluyendo nuevos ingredientes como la leche y la canela. También innovaron en los procesos de producción e impulsaron nuevas presentaciones: líquidas, en polvo, mezclas con diversos ingredientes, hasta llegar al chocolate sólido en barra. Los hallazgos se clasificaron de acuerdo a las formas de difundir y comercializar el producto, en tres grandes periodos: Pre-Colonia, Colonia y Post-Colonia. La primera etapa se caracterizó por el intercambio y comercio; la segunda por la innovación y distribución y la tercera, por el uso del marketing agroindustrial y marketing promocional. Así, se aprecia que el chocolate ha formado parte esencial en la vida de los mexicanos, de su historia, cultura y gastronomía. Su presencia desde tiempos prehispánicos a la época actual ha pasado por un proceso de transformación no solo en su forma de fabricación, sino en el consumo y la difusión del mismo. Este producto es de suma importancia económica para las empresas que se dedican a su fabricación y comercialización en todo el mundo. Las tres grandes líneas son: chocolate para mesa, chocolate Premium y chocolate-golosina, la más conocida y la de mayor posicionamiento a nivel global. El marketing del chocolate tiene especial importancia por la competitividad de los mercados donde participa.

PALABRAS CLAVE: Chocolate. Comercialización. Historia. 
EL CHOCOLATE, DE BEBIDA DE NOBLES A GOLOSINA DE TODOS

\section{ABSTRACT}

This document is the result of qualitative research with an inductive approach in which findings were taken from literary review. The research followed a strictly descriptive order; the procedure was oriented to develop a narrative explanation on the historical process of the diffusion and commercialization of the chocolate from the pre-hispanic to the present time. Several authors gave an account of the transformations of cacao, from the original concept of mixed drink with different spices, which arrived in Spain and from there to Europe, where they added value, including new ingredients such as milk and cinnamon, innovating it with liquid versions, powder, until the solid chocolate bar. The findings were classified according to the ways of disseminating and marketing the product, in three large periods: Pre-Cologne, Cologne and Post-Cologne. The first stage was characterized by trade and commerce; the second for innovation and distribution and the third for the use of agro-industrial marketing and promotional marketing. Chocolate has been an essential part of mexicans, their history, culture and gastronomy from pre-hispanic times to the present time. This product is of great economic importance for the companies that are dedicated to its manufacture and commercialization all over the world. The three main lines are: table chocolate, Premium chocolate and chocolate-candy, the best known and the highest positioning globally. Chocolate marketing is especially important because of the competitiveness of the markets where it participates.

KEYWORDS: Chocolate. Commercialization. History. 
EL CHOCOLATE, DE BEBIDA DE NOBLES A GOLOSINA DE TODOS

\section{Marco teórico}

Las primeras aproximaciones al marketing actual fueron establecidas a través de la comercialización, cuyo primer curso se dictó en Alemania en 1898, cuatro años después se hizo lo propio en los Estados Unidos de América donde se impartió el seminario: The distributive and regulative industries of the United States. Tiempo después, entre 1910 y 1920 se empezaron a crear los primeros centros de investigación en marketing y su desarrollo conceptual (Bartels, 1988).

El marketing se asocia con periodos de abundancia económica, donde se privilegia a la distribución e innovación de productos. Sus primeras conceptualizaciones fueron relacionadas con la distribución, intercambio y comercio (Mercado, 2006). Según Kotler y Armstrong (2001), comercialización se refiere a la introducción de un nuevo producto al mercado; en tanto que para Rodríguez (2005), es una actividad comercial que se realiza para vender y distribuir lo ya producido. De acuerdo con Stanton, Etzel y Walker (2000), comercializar es una actividad fundamental de ventas.

Posterior a la era de la comercialización, el marketing se vislumbró como un proceso de distribución. Así lo asumió a la Asociación Americana de Marketing, en 1960, cuando la conceptualizó como la realización de actividades empresariales que dirigen el flujo de bienes y servicios desde el productor hasta el consumidor o usuario (AMA, 1960). Después se le dio un énfasis más integral y se le difundió como el proceso de planificación y ejecución de la concepción, precio, comunicación y distribución de ideas, productos y servicios para crear intercambios que satisfagan a los individuos y a los objetivos de la organización (AMA, 1985).

Sin duda, los cambios en la orientación del marketing han sido continuos y se han caracterizado por experimentar diferentes enfoques, tales como la orientación a la producción, ventas, productos, mercados y clientes (Camacho, 2015). Según Kotler (2001), el marketing tiene como tarea primordial la creación, promoción y entrega de bienes y servicios a los consumidores y a los negocios.

Aunque en la actualidad la comercialización y el marketing se complementan; el primero ha pasado a formar parte del segundo. Esto depende del producto, de los segmentos y las industrias y mercados en donde participa la empresa. El marketing es esencialmente una forma de detectar y satisfacer ciertas necesidades de la gente (Bartel, 1988). Cuando 
EL CHOCOLATE, DE BEBIDA DE NOBLES A GOLOSINA DE TODOS

tiene como meta al cliente, entonces se deben diseñar y ejecutar acciones estratégicas para

Conocer, persuadir y mantener segmentos de mercado satisfechos. Este enfoque exige una planeación bien definida de corto y mediano plazo, tomando en consideración que los estilos de vida y el comportamiento de compra de los consumidores es altamente cambiante y que las opciones que tendrá para adquirir el producto o servicio en cuestión, también serán amplias (Camacho y Andrade, 2015, p. 18).

El marketing hoy día se le asume como una actividad desarrollada por instituciones mediante procesos para crear, comunicar, entregar e intercambiar ofertas que tengan valor para los consumidores, clientes, socios y sociedad en general (AMA, 2013). Sin embargo, debe tomarse en cuenta que la dinámica global ha llevado a una diversificación notoria de las especialidades del marketing. Para fines de este artículo sólo consideraremos al marketing agroindustrial, donde el cliente es clave, pero también lo es la cadena productiva. De tal manera que si conocemos las tendencias actuales y las futuras, el fabricante puede adelantarse a los requerimientos de sus clientes (Rodríguez, 2005), donde La creación de valor está basada en los elementos intangibles (Lovelock y Wirtz, 2009).

De acuerdo con Alejandro Jáuregui (2001), el marketing agroindustrial se refiere al comportamiento de los bienes y servicios del sector agrario, a la promoción de los productos agrícolas a nivel mundial. En el marketing agroindustrial lo más importante es el producto, la materia prima y los insumos con los que son fabricados. La agroindustria transforma productos primarios provenientes de la agricultura, ganadería, recursos forestales y pesca; añadiendo valor para ofrecer productos de calidad en los mercados que atiende, mediante la integración vertical del proceso (Camacho, 2012). De acuerdo con la Secretaría de Agricultura, Ganadería, Desarrollo Rural, Pesca y Alimentación (SAGARPA), el sector agroindustrial es parte de la industria manufacturera en su División de alimentos procesados y bebidas, que a su vez se conforma de 12 sub-industrias: carnes y lácteos, frutas y legumbres, molienda de trigo, molienda de nixtamal, beneficio y molienda de café, azúcar, aceites y grasas comestibles, alimentos para animales, otros productos alimenticios, bebidas alcohólicas, cerveza y malta, refrescos y aguas gaseosas (SAGARPA, 2013). El marketing agroindustrial tiene como actividad principal a la 
EL CHOCOLATE, DE BEBIDA DE NOBLES A GOLOSINA DE TODOS

comercialización debido al tipo de productos que oferta, usualmente con altos componentes de innovación y por lo tanto diferenciados.

El presente trabajo fue desarrollado con enfoque inductivo; basado en la técnica de revisión documental y tomando en cuenta que en este tipo de estudios se producen datos escritos en diversos documentos, donde el investigador construye el conocimiento (Ibañez, 2002). La investigación siguió un orden estrictamente descriptivo, el procedimiento fue orientado a desarrollar una explicación narrativa sobre el proceso histórico de la difusión y comercialización del chocolate desde la época prehispánica a la actual.

\section{Pre-Colonia, Colonia y Post-Colonia}

Los principales hallazgos de este estudio muestran que la comercialización del chocolate a través de la historia ha pasado al menos por tres grandes periodos: pre-colonia, colonia y post-colonia (Ver figura 1 ).

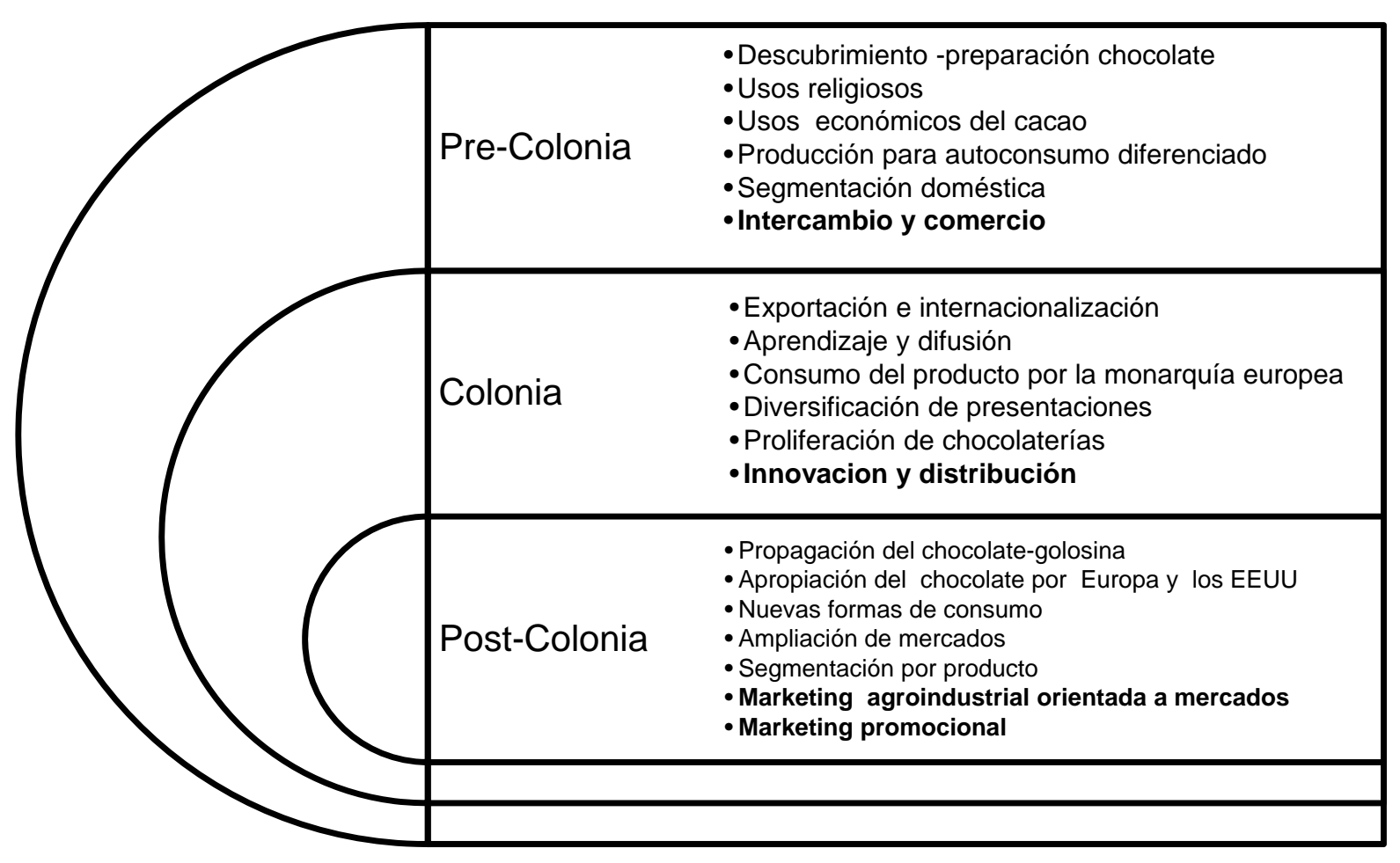

Figura 1. Proceso de difusión y comercialización del Chocolate.

Fuente: Elaboración propia. 
EL CHOCOLATE, DE BEBIDA DE NOBLES A GOLOSINA DE TODOS

\section{a. Periodo pre-Colonial}

El chocolate indudablemente es de origen mexicano, el cacao su materia prima, ha sido estudiado desde diferentes vertientes (Camacho, 2017). Apreciado como un alimento muy preciado (Colmenero de Ledesma, 1631); se le atribuyeron propiedades medicinales (López y López, 1875) y referido como ofrenda a deidades prehispánicas (Aranda, 2014). También es muy conocida su representatividad como moneda de cambio y tributo durante los cacicazgos indígenas y españoles (Hardwich, 2011).

El cacao es conocido como una materia prima agrícola, base de subproductos tales como la pasta, manteca y polvo de cacao; así como de productos finales muy conocidos como los chocolates, bombones, bebidas achocolatadas (Quintero, 2015). Los primeros cultivos conocidos corresponden a la cultura maya (500 a. C.), cuyo nombre proviene de la palabra "cac", que significa rojo, en referencia al color de sus frutos y "cau", que expresa la idea de fuerza y fuego. Los aztecas lo denominaban "cacahuat". Las culturas mayas, toltecas y aztecas le atribuían origen divino (dios Quetzalcóatl) y por ello emplearon el nombre de xocoatl (chocolate) para referirse a la bebida aromática que se obtenía con ellos y a la que atribuían un efecto reconstituyente e incluso afrodisiaco (Ramírez, 2017).

En 1753 el científico sueco Carl von Linné (conocido como Linnaeus) nombró científicamente al árbol del cacao como Theobroma cacao (Coe, Michael, 2000; Camacho, 2014). Theobroma, el nombre genérico proveniente del griego, se puede traducir como "comida o alimento de los dioses" (Ver figura 2). Aunque Linnaeus se dice, era apasionado por el chocolate, se asume que éste habría revisado los primeros escritos españoles que describían las creencias mayas y aztecas de que el cacao era un regalo de los dioses (Kommunarka, 2011). 

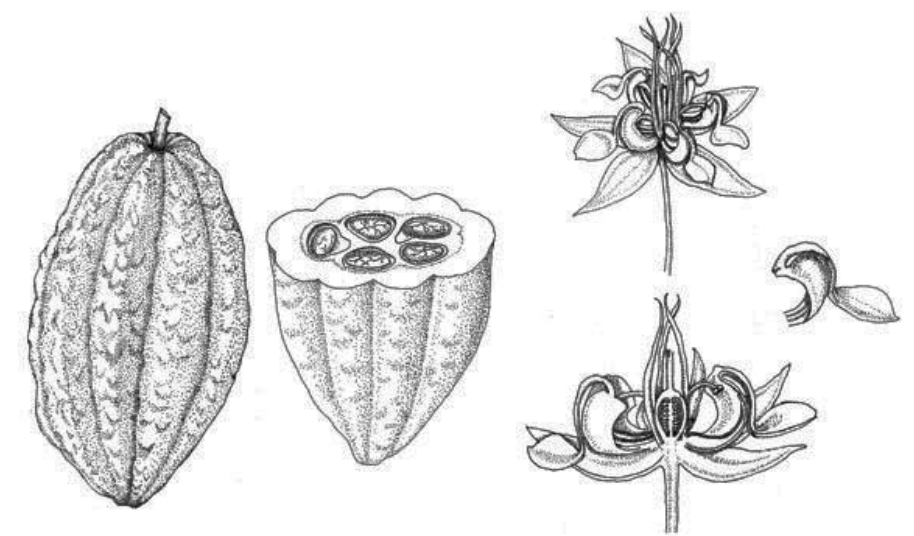

Figura 2. Theobroma cacao.

Fuente: Mexicocolore (2014).

Los estudiosos del cacao y el chocolate, estiman gran representatividad de estos productos en las culturas aztecas y mayas donde su uso y difusión fue muy significativa. Si bien no se sabe con exactitud cuándo y quiénes fueron los primeros en convertir las semillas de cacao en una bebida reconstituyente, hay evidencia de que los antiguos Olmecas de México prepararon lo que se podría suponer como el primer chocolate. El cual incluía entre sus ingredientes: granos de cacao molidos en piedras y cocidos en agua, mezclada con miel de maguey, harina de maíz, achiote y vainilla (El Universal, 2008). Después de los Olmecas, este manjar se extendió a las civilizaciones maya y azteca, en donde fue considerado como el alimento de los dioses (SAGARPA, 2017).

En México, el chocolate se propagó como una bebida, que al igual que otras que tenían como ingrediente principal al cacao, eran consideradas de gran valor entre los diferentes grupos indígenas. Se puede decir que entre los antiguos mexicanos, la educación en el consumo del chocolate inicia al considerársele como una bebida alimenticia, con propiedades favorables para la salud, como fuente energética, preparada con materias primas naturales: cacao, orejuela, pimienta y súchel (Ruz, 2016). En un primer momento fue un producto privilegiado y consumido por la realeza indígena mexicana y después, para la hispana. Para los aztecas existían dos bebidas fundamentales: el octli, una especie de vino nativo de la región (pulque), y el xocoátl. Esta última reservada para la élite azteca y los guerreros, ni siquiera los sacerdotes lo consumían ya que tenían que llevar una vida de austeridad y penitencia (Cervantes, 2012). 
EL CHOCOLATE, DE BEBIDA DE NOBLES A GOLOSINA DE TODOS

En su último viaje al Nuevo Continente, Cristóbal Colón desembarcó el 15 de agosto de 1502 en la isla de Guanaja, donde se encontró con comerciantes aztecas que le ofrecieron semillas de cacao, además de que le mostraron como preparar la bebida (Ver figura 3). Después de probarla, Colón y su grupo no sintieron agrado por el sabor del xocoátl. Sin embargo, retornaron a España con algunas de las almendras de cacao sin conocer su valor económico y comercial que este producto alcanzaría en los siglos venideros (Aguirre, 2005).
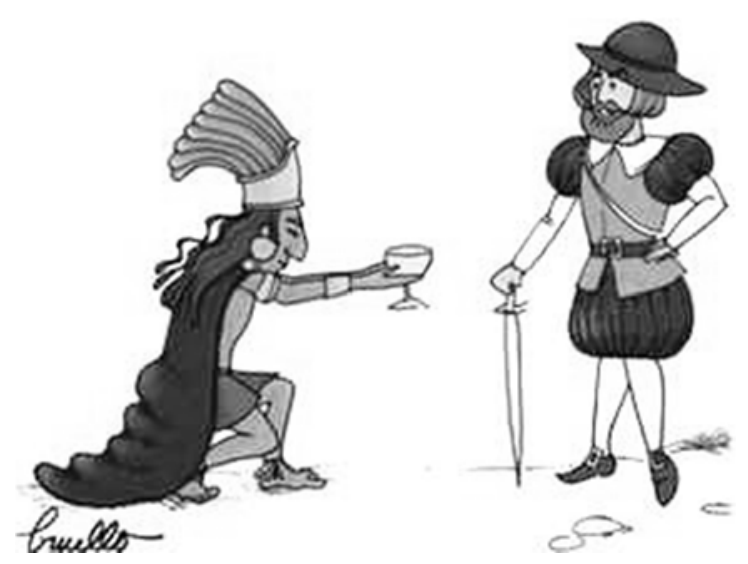

Figura 3. Aztecas comparten cacao con europeos.

Fuente: Arindabo, 2017.

\section{b. Periodo Colonial}

Jerónimo de Aguilar, un náufrago español asentado en Yucatán por varios años, aprendió las propiedades y la formas de preparación del cacao. En 1519 cuando Hernán Cortés arribó a esas tierras, pasó a formar parte de su grupo, aprovechando que hablaba lengua maya, fue útil como intérprete. Cuando se instaló en la capital mexicana, se dedicó a probar nuevas formas de preparar este producto y logró una pasta de cacao. Después de este hecho estimó necesario mezclar un poco de miel para atenuar el amargo tan pronunciado del fruto, que se cultivaba de manera silvestre. Esta innovación la dio a probar a sus compatriotas, el cual fue bien recibido y lo consideraron un alimento gustoso y aromático. Fue tal la aceptación que empezaron a demandar grandes porciones, Jerónimo producía pero guardaba celosamente su secreto. Los primeros dos años de su comercialización la pasta se consumía cruda, pero después, decidieron hervirla en agua, como si fuera café. Esta bebida llegó a sustituir otros alimentos que se consumían en el 
EL CHOCOLATE, DE BEBIDA DE NOBLES A GOLOSINA DE TODOS

desayuno. A esta pasta hervida en agua, se le dio el nombre de chocolate (López y López, 1875, p. 20, citado por Camacho, 2017).

Entre todas la bebidas elaboradas a base de cacao, el chocolate fue el que llamó más la atención de los conquistadores, quienes lo portaron a España y de allí a las principales ciudades de Europa (Camacho, 2014). Los hispanos conocieron el chocolate en la mesa de Moctezuma, ya que era una de las bebidas predilectas del emperador (Aguirre, 2005).

Ya en Europa, fue Carlos I de España, quien tuvo la idea de mezclar el cacao con azúcar, canela y vainilla y de ese modo obtuvo una golosina más del agrado de los paladares europeos, naciendo así el chocolate moderno (Aguirre, 2005). Después de su ingreso y éxito comercial en este país, el chocolate llegó a Francia, donde se presume que entró por sus propiedades curativas a través del Cardenal de Lyon: Alphonse Richelieu quien lo utilizaba para aliviar sus dolencias, con el deceso de este personaje, asumió el puesto Giulio Mazarini, quien era amante del chocolate. Fue él quien otorgó a David Challiou el privilegio exclusivo de preparar y vender chocolate en todo el reino (Kommunarka, 2011).

A finales del siglo XVI los ingleses redactaron escritos sobre el chocolate y las nuevas formas de consumirlo; agregándole huevos y leche en lugar de agua. Este producto ocupaba un lugar preponderante en la vida de los europeos, entre los quienes surgieron diversas leyendas (Meraz, 2014). Hasta principio del siglo XIX el chocolate existió solamente como bebida, pero en 1819, el suizo Francois-Louis Cailler, creó la primera barra de chocolate duro del mundo (Rubín, 2017). Hacia 1825 Daniel Peter inventó el chocolate con leche. Este ingrediente era suministrado por el empresario Henri Nestlé. En 1929 la compañía de Nestlé se fusionó con la de Peter, Kohler y Cailler, juntos llegaron a ser el mayor fabricante del chocolate suizo (Nestlé, 2016).

El chocolate no solamente conquistó Europa. Las Filipinas, colonizada por los españoles en 1543, fue otro de los muchos países que se vieron envueltos en la euforia por el chocolate; estas islas eran la fuente de chocolate de misioneros jesuitas y de los comerciantes portugueses. Para los habitantes de Filipinas beber chocolate se convirtió en una tradición (Coe, 1999). 
EL CHOCOLATE, DE BEBIDA DE NOBLES A GOLOSINA DE TODOS

El chocolate también arribó a América del Norte a través de altos funcionarios que lo llevaron a lugares como Virginia y Massachuset de los Estados Unidos de América. En este país, fue Milton Snavely Hershey propietario de un negocio de caramelos, lo vendió para dedicarse y llegar a ser el principal comercializador de chocolate, hasta ser considerado como "el Henry Ford de los fabricantes de chocolate". Hershey se preocupó de la calidad de sus productos y contrató nutriólogos para reforzar las propiedades alimenticias de sus chocolates (Coe, 2000).

\section{c. Post-Colonia}

Al presente, el mercado mundial privilegia la comercialización del chocolate en su presentación de golosinas y barras, el chocolate para mesa en menor proporción. Por su parte, el chocolate Premium es un chocolate artesanal pero refinado, se trata de un producto más puro (Rivera, 2016).

Es muy apreciado por sus propiedades, aroma inigualable, sabor exquisito y de valor energético altamente reconocido. Estas características provienen de las propiedades del cacao, y no de los demás ingredientes. Por ello, existe la tendencia de los grandes fabricantes por dar mayor importancia a la variedad y tipo de cacao que se utiliza en la preparación de sus chocolates. Se trata de una distinción de este tipo de productos, los cuales al ser más finos, son más valorados por los consumidores, quienes cada día aprecian más el sabor amargo y fuerte del cacao, con contenidos de altos porcentajes del grano (Parizot, 2010, p. 30).

Se observa que la diversificación y presentaciones del chocolate que hoy se encuentran en el mercado, es el resultado de un legado cultural de mexicanos, españoles, italianos, franceses, suizos, suecos, alemanes, austriacos, holandeses, ingleses y americanos. Todos ellos aportaron su creatividad y tecnologías hasta lograr un producto de calidad mundial, con gran significancia cultural y gastronómica (Camacho, 2017).

Con estas perspectivas, tres grandes líneas de chocolate prevalecen en el mercado: chocolate tradicional (para mesa), chocolate golosina y chocolate gourmet o Premium (vean to bar). En general, sus principales atributos pueden clasificarse en tangibles e intangibles, donde la calidad real debería ser convergente con la calidad percibida (ver tabla 1). 
EL CHOCOLATE, DE BEBIDA DE NOBLES A GOLOSINA DE TODOS

Tabla 1

Componentes tangibles e intangibles del chocolate

\begin{tabular}{ll}
\hline Tangibles & Intangible \\
\cline { 2 - 3 } Ingredientes & $\begin{array}{l}\text { Marca (conciencia, } \\
\text { aceptación y } \\
\text { preferencia) }\end{array}$ \\
Sabor & Prestigio de la empresa \\
Calidad real del & Calidad percibida en el \\
producto & producto \\
Diseño del Empaque & Publicidad \\
Color & Innovación \\
Presentaciones & $\begin{array}{l}\text { Investigación y } \\
\text { desarrollo }\end{array}$ \\
Precio & Interactividad \\
Accesibilidad al & Celebridades \\
\hline
\end{tabular}

Fuente: Camacho (2015).

El proceso histórico del chocolate ha pasado del marketing de distribución al marketing de comunicaciones integradas, también conocida como marketing promocional. Es decir, las empresas fabricantes y comercializadoras de chocolate, si bien tienen en cuenta las necesidades del mercado y de sus segmentos; su mayor inversión se encuentra en la publicidad, promoción de ventas, patrocinios y uso de personalidades para posicionar y mantener altas participación de mercados (Camacho, 2015). 
EL CHOCOLATE, DE BEBIDA DE NOBLES A GOLOSINA DE TODOS

\section{CONCLUSIONES}

El chocolate a través del tiempo ha formado parte esencial en la vida de los mexicanos, de su cultura y de su economía. En este escenario, Tabasco, México; es la tierra donde olmecas y mayas convergieron para hacer del cacao y el chocolate un "alimento" de gran virtud, al cual le conferían atributos de fortaleza física para los guerreros y propiedades curativas (Camacho, 2017).

La revisión del estado del arte de la comercialización histórica del chocolate, muestra que este producto pasó de una producción de autoconsumo como bebida para las élites prehispánicas a una fabricación exclusiva para la realeza europea. Región donde el producto original fue transformado en otras presentaciones, con nuevos ingredientes y formas; como es el caso del chocolate-golosina, conocido y comercializado en todo el mundo.

Asimismo, se aprecia una tendencia creciente por consumir chocolate artesanal pero fino. Esta es la categoría de chocolates Premium, para el cual las empresas fabricantes han identificado nuevos nichos a partir de combinaciones del cacao con insumos exóticos y con tendencias de crecimiento diferenciado. Para estimular la demanda, las empresas utilizan actualmente el marketing agroindustrial en combinación con el marketing promocional; de esta última especialidad del marketing, se sugieren nuevas indagatorias que permitan una mejor comprensión de su uso en el mercado del chocolate.

\section{REFERENCIAS BIBLIOGRÁFICAS}

Aguirre, Manuel (2005). El cacao...orgullosamente mexicano. México. Recuperado de: http://mexicomaxico.org/dadivas/cacao.htm

AMA. Common language in marketing. http://marketing-dictionary.org/ama

Aranda, Lucía (2014). El uso de cacao como moneda en la época prehispánica y supervivencia en la época colonial, p. 1440.

Arindabo (2017). Las pascuas. Recuperado de: http://arindabo.blogspot.mx/2017/04/laspascuas.html 
EL CHOCOLATE, DE BEBIDA DE NOBLES A GOLOSINA DE TODOS

Bartel, R. (1988). The history of marketing thought.

Boleaga, Ernesto. (2011).Tendencias gastroculinarias. Revista Gourmet, Aniversario 25. México.

Cacao, México. (2015). Historia del cacao. Recuperado de: http://www.cacaomexico,org/

Camacho, M. (2012). El marketing en la agroindustria. Revista Hitos de Ciencias Económico Administrativas 18(51), 52-54.

Camacho, M. (2014). Chocolate del edén para el mundo. Historia, gastronomía y turismo. Villahermosa, Tabasco, México: Consejo Coordinador Empresarial-Universidad Juárez Autónoma de Tabasco.

Camacho Gómez, M. \& Andrade Torres, J. (2015). Mercadotecnia en perspectiva: Reflexiones y casos. México: Trillas.

Camacho Gómez, M. (2015). Marketing del Chocolate en México. Vinculatégica Año 1, No. 1. 1er. Congreso Internacional de Investigación en Escuelas y Facultades de Negocios: Vinculación Estratégica en las Organizaciones. FACPYA UANL, Monterrey, N.L. Junio 2015.

Camacho Gómez, M. (2017). Chocolate historia, arte, cultura y marketing: tendencias mundiales. México: Editorial Laberinto-UJAT.

Cervantes, Jair, et al. (2012) Xocolatl: antes alimento de los dioses, y ahora... Revista de divulgación científica y tecnológica de la universidad veracruzana. México.

Coe, M. D. (1999). La verdadera historia del chocolate. Editorial: S.L. Fondo de cultura económica de España.

Coe, M.D. (2000). Kakaw. Recuperado de: http://www.popolvuh.ufm.edu/

Museo Kakaw, (2015). Origen botánico prehistórico y sus antecedentes. Recuperado de: http://www.kakaw.org/ .

Hershey, Company (2017). Nuestra historia. Recuperado de www.thehersheycompany.com. 
EL CHOCOLATE, DE BEBIDA DE NOBLES A GOLOSINA DE TODOS

Ibáñez, Jesús (1992). La guerra incruenta entre cuantitativistas y cualitativistas: Las ciencias sociales en España: Historia inmediata, críticas y perspectivas. Universidad Complutense de Madrid.

Instituto Mexicano de la Propiedad Industrial (2016). La importancia del chocolate. Recuperado de: http://www.gob.mx/impi/articulos/el-cacao

Jáuregui, Alejandro (2001). Marketing agroindustrial. Recuperado de: https://www.gestiopolis.com/marketing-agroindustrial/

Kommunarka (2011). About chocolate: History of Chocolate. Recuperado de: http://www.eng.kommunarka.by

Kotler, P. Armstrong, G. (2003). Fundamentos de marketing. México: Pearson Educación.

Lovelock, Ch., Wirtz, J. (2009). Marketing de servicios. Personal, tecnología y estrategia. Sexta Edición. México: Pearson/Prentice Hall.

Mejía, L. et al (2014). Tendencias gastronómicas. Universidad Autónoma del Estado de México. México. p. 30.

Meraz, L.M., (2014). Beneficios del chocolate para la salud: ¡Sé más inteligente y vive más! Recuperado de: http://www2.esmas.com/salud/nutricion.

Mercado H. S- (2006). Mercadotecnia Programada. Principios y aplicaciones para orientar la empresa hacia el mercado. México: Limusa-Grupo Noriega Editores

Mexicocolore (2014). History and use of cacao. Recuperado de http://www.mexicolore.co.uk/maya/chocolate/history-and-uses-of-cacao

Nestlé, (2016), Línea de Tiempo de Nestlé: 1866-actualidad. Recuperado de: http://www.nestle.com.ar .

Parizot Wolter, A. (2010). Cacao en Tabasco: el origen. 1er. Festival del Chocolate Tabasco. Villahermosa, México: Secretaría de Turismo.

Quintero Rizzuto, M.L. (2015). Tesis doctoral, "Productos básicos agrícolas y desarrollo: producción y comercialización de cacao en Venezuela". 
EL CHOCOLATE, DE BEBIDA DE NOBLES A GOLOSINA DE TODOS

Ramírez, Mario (2017). El cacao, un cultivo milenario que en México tiene una tendencia a desaparecer. Dirección de comunicación de la Ciencia. Universidad Veracruzana, México.

Revista Digital Universitaria, (2015). Encuentros con el chef chocolatero José Ramón Castillo. Recuperado de: http://www.revista.unam.mx/vol.12/num4/art39/art39.pdf.

Rivera, F. (2016). ¿Qué es el Chocolate Bean to Bar? Recuperado de https://experiencias.wherelunch.com/2016/05/26/que-es-el-chocolate-bean-to-bar/

Rodríguez, G. A. (2005). Agronegocios: de la comercialización al marketing.

Rubín, María José, (2017). Francois-Louis Cailler. La historia del chocolate. Recuperado de: http://sobresuiza.com.

Ruiz Olabuénaga, J.I. (1996). Metodología de investigación cualitativa. Bilbao: Deusto.

SAGARPA (2017). ¡Por amor al chocolate! Recuperado de https://www.gob.mx/sagarpa/articulos/por-amor-al-chocolate?idiom=es

SAGARPA (2013). Monitor Agroeconómico e Indicadores de la Agroindustria. Recuperado de http://www.sagarpa.gob.mx/agronegocios/Documents/MonitorNacionalMacro_nv.pdf

Samaja, J. (1993). Epistemología y metodología. Elementos para una teoría de la investigación científica. Buenos Aires: Editorial universitaria.

Universal, (Julio 29, 2008). Consumían olmecas chocolate hace 3 mil años. Recuperado de http://archivo.eluniversal.com.mx/notas/526113.html 